\title{
Téoros
}

Revue de recherche en tourisme

\section{La réorganisation municipale et ses effets prévisibles sur l'offre touristique décentralisée au Québec}

\section{Danielle Pilette}

Volume 19, numéro 3, automne 2000

Organisations touristiques en mutation

URI : https://id.erudit.org/iderudit/1071737ar

DOI : https://doi.org/10.7202/1071737ar

Aller au sommaire du numéro

Éditeur(s)

Université du Québec à Montréal

ISSN

0712-8657 (imprimé)

1923-2705 (numérique)

Découvrir la revue

Citer cet article

Pilette, D. (2000). La réorganisation municipale et ses effets prévisibles sur l'offre touristique décentralisée au Québec. Téoros, 19(3), 10-14.

https://doi.org/10.7202/1071737ar d'utilisation que vous pouvez consulter en ligne. 


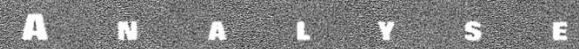

\section{LA RÉORGANISATION MUNICIPALE ET SES EFFETS PRÉVISIBLES SUR L'OFFRE TOURISTIQUE DÉCENTRALISÉE Au Québec}

\section{Danielle Pilette}

Depuis une quarantaine d'années, parallèlement aux effets de la "Révolution tranquille ", le secteur local au Québec a révisé son offre de services de façon importante en y incluant progressivement des services aux personnes par opposition aux seuls services en réseaux et aux services à la propriété qui constituaient sa mission traditionnelle (adduction d'eau, égouts, gestion des déchets, réseau routier, protection contre les incendies, police, etc.).

Même si, en part relative, les services aux personnes représentent toujours une activité marginale du secteur local, il n'en demeure pas moins que les sommes dépensées y sont très significatives, compte tenu que les dépenses totales des municipali- tés locales s'élevaient à plus de huit milliards de dollars en 1996 (ministère des Finances, 1996 : I) et qu'elles sont estimées à près de dix milliards actuellement. Les montants que le secteur local alloue spécifiquement aux aménagements et au

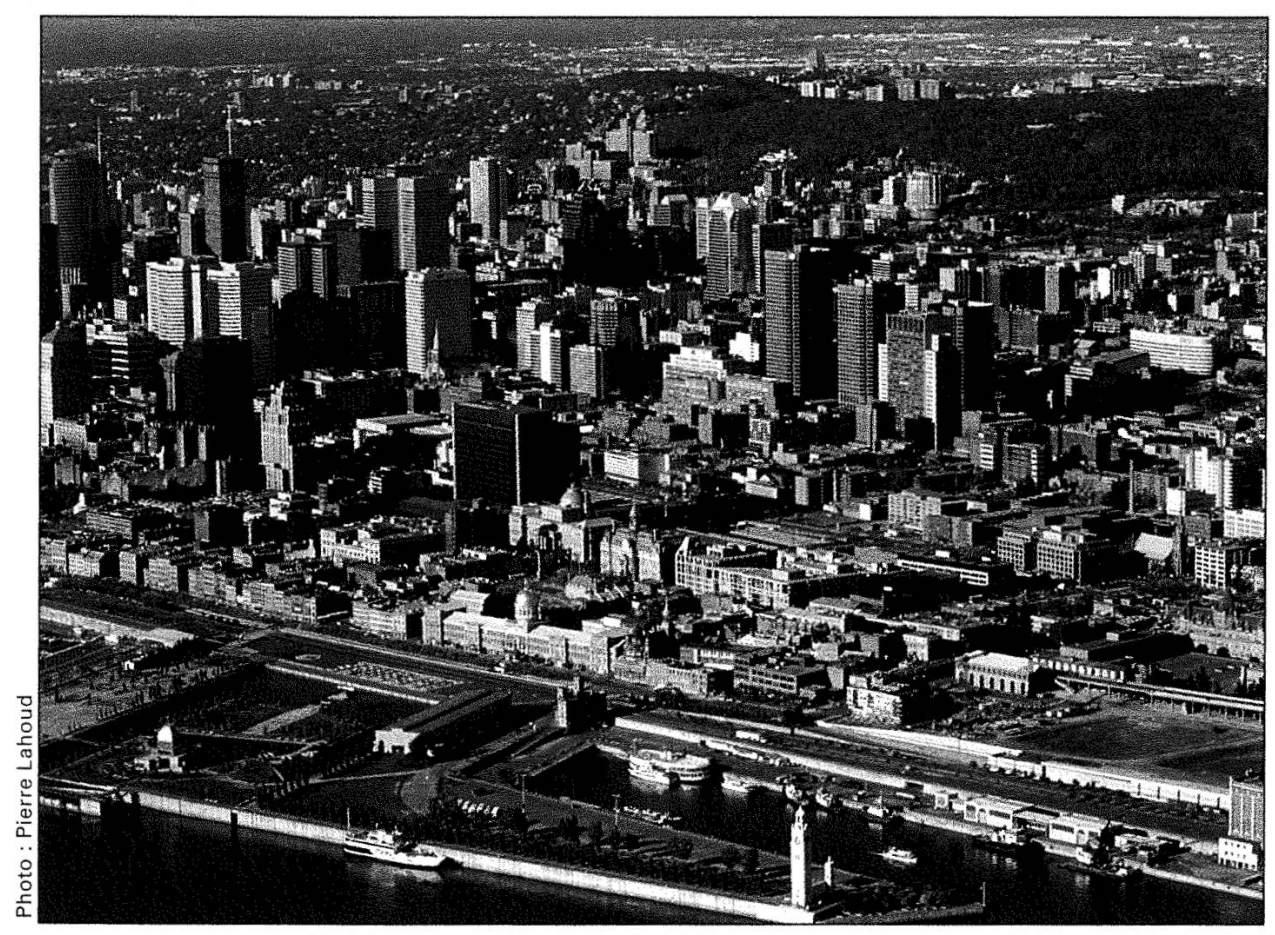

Le centre-ville de Montréal développement touristiques sont d'autant plus difficiles à cerner avec précision qu'ils sont fréquemment compris dans diverses enveloppes de mise en valeur du territoire, de développement et de promotion économiques, de loisirs et de culture.

L'évolution des municipalités locales dans le secteur des services aux personnes physiques ou morales, comme les entreprises et les organismes, résulte moins d'un phénomène de décentralisation que d'un processus de revalorisation du pouvoir municipal.

\section{L'OPPORTUNITÉ DES DISPARITÉS ET LE POUVOIR LOCAL : PERSPECTIVE 1980-2000}

Au plan juridique, les municipalités locales bénéficient de délégation de pouvoirs provinciaux notamment par des lois et des chartes constitutives qui leur permettent d'assumer leurs fonctions. En ce sens, elles constituent des instances décentralisées. Cependant, ce type d'assujettissement, juridique, est largement contrebalancé, particulièrement depuis les vingt dernières années, par leur autonomie financière et leur manière de l'exercer aux fins de leur mission d'allocation.

Dans un document de référence préparé par le ministère des Affaires municipales et déposé par le Secrétariat des conférences socio-économiques à la conférence Québec-municipalités, tenue à Québec en juin 1978 , le gouvernement affirmait « retenir l'option de l'autonomie locale » après de nombreuses consultations et études. « L'autonomie locale implique qu'il ap- 
partient à chaque collectivité locale de déterminer la nature et la qualité des services ainsi que les taxes requises pour les financer. Cela suppose qu'il n'y ait pas de nécessité d'égalisation des services à l'échelle du Québec et que certains écarts de ressources et de fardeau fiscal puissent exister entre les municipalités » (Conférence Québec-municipalités, 1978 : 26).

La situation de diversité du type et de la quantité de services offerts que l'on note aujourd'hui au palier local relève donc bien moins de causes conjoncturelles, de con- flits de personnalités politiques et d'esprit de clocher - comme l'évoque souvent la tradition populaire - que d'un phénomène structurel prévisible et accepté pour les besoins d'une concertation formalisée il y a plus de vingt ans par un gouvernement issu du même parti que celui qui est actuellement au pouvoir.

On constate de plus que l'autonomie financière locale est presque absolue pour les municipalités de plus de 5000 habitants (tableau 1). En effet, les municipalités de cette taille reçoivent moins de $2 \%$ de leurs recettes des transferts gouvernementaux.

\section{L'OFFRE TOURISTIQUE DANS UNE ORIENTATION LOCALE AMÉNAGISTE : CAP SUR LE PARC IMMOBILIER}

En particulier dans les régions métropolitaines aux marchés segmentés, plusieurs municipalités privilégient une approche aménagiste qui vise non seulement la notoriété, mais aussi la valeur du parc immobilier. Bien que l'exercice de responsabilités locales en matière de tourisme soit complètement facultatif, on ne s'étonnera pas que les municipalités à orientation aménagiste tentent de recruter des inter-

\section{TABLEAU 1}

Recettes des municipalités locales selon la taille - 1996

\begin{tabular}{|c|c|c|c|c|c|c|}
\hline & \multicolumn{2}{|c|}{ Moins de 5000 habitants } & \multicolumn{2}{|c|}{5000 à 49999 habitants } & \multicolumn{2}{|c|}{50000 habitants et plus } \\
\hline & en $M \$$ & en $\%$ & en $\mathbf{M \$}$ & en $\%$ & en MS & en $\%$ \\
\hline \multicolumn{7}{|l|}{ Taxes foncières } \\
\hline Taxe générale & 563 & 49,1 & 1392 & 49,2 & 2053 & 48,6 \\
\hline Autres & 20 & 1,7 & 106 & 3,7 & 90 & 2,1 \\
\hline Total des taxes foncières & 584 & 50,9 & 1498 & 52,9 & 2143 & 50,8 \\
\hline Tarification pour services municipaux & 194 & 16,9 & 433 & 15,3 & 457 & 10,8 \\
\hline $\begin{array}{l}\text { Taxe et surtaxe foncière sur les } \\
\text { immeubles non résidentiels et taxe } \\
\text { d'affaires }\end{array}$ & 31 & 2,7 & 243 & 8,6 & 520 & 12,3 \\
\hline $\begin{array}{l}\text { Compensations tenant lieu de } \\
\text { taxes - réseaux des entreprises } \\
\text { TGE* }\end{array}$ & 81 & 7,1 & 116 & 4,1 & 166 & 3,9 \\
\hline $\begin{array}{l}\text { Compensations tenant lieu de } \\
\text { taxes - immeubles } \\
\text { gouvernementaux et autres } \\
\text { immeubles }\end{array}$ & 30 & 2,6 & 112 & 3,9 & 271 & 6,4 \\
\hline Autres recettes de source locale & 99 & 8,6 & 387 & 13,7 & 588 & 13,9 \\
\hline Total des recettes de source locale & 1018 & 88,8 & 2788 & 98,4 & 4145 & 98,2 \\
\hline Transferts & & & & & & \\
\hline Inconditionnels & 17 & 1,5 & 9 & 0,3 & 41 & 1,0 \\
\hline Conditionnels & 111 & 9,7 & 35 & 1,2 & 36 & 0,9 \\
\hline Total des transferts & 127 & 11,1 & 44 & 1,6 & 77 & 1,8 \\
\hline Total des recettes & 1146 & 100,0 & 2832 & 100,0 & 4222 & 100,0 \\
\hline $\begin{array}{l}\text { Compte de taxes moyen - } \\
\text { résidence unifamiliale (en dollars) } \\
\text { Richesse foncière uniformisée per capita } \\
\text { (en dollars) }\end{array}$ & $\begin{array}{r}850 \\
38585\end{array}$ & - & $\begin{array}{r}1850 \\
45405\end{array}$ & - & $\begin{array}{r}2390 \\
47083\end{array}$ & - \\
\hline
\end{tabular}

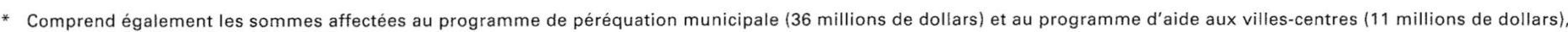
financés à même les recettes de la compensation TGE.

Note: Le total peut ne pas correspondre à la somme des éléments en raison de l'arrondissement des données.

Source : ministère des Affaires municipales, 1996 
venants touristiques majeurs et des projets d'envergure, avec parc immobilier et attractions qui apportent une assiette de valeur considérable.

Si l'évolution de leur parc hôtelier est suivie avec attention par les grandes villes, de grands projets récréotouristiques soutiennent aussi la notoriété et la richesse foncière de certaines municipalités de leurs régions limitrophes, par exemple des Laurentides et des Cantons de l'Est. Plus on privilégie l'approche aménagiste, plus on raffine ses avantages concurrentiels et plus on vise à instaurer ses propres structures de promotion - soit économiques, soit spécifiquement touristiques - qui peuvent toujours dupliquer celles qui sont mises en commun dans les territoires des communautés ou des municipalités régionales de comté.

Même en régions périmétropolitaines, on observe la prospérité de territoires largement dédiés à la villégiature et au tourisme. Ainsi, alors que le tableau 1 montre une richesse foncière uniformisée per capita variant de 38585 \$ à $47083 \$$ en 1996 selon la taille des municipalités du Québec, on trouve des résultats beaucoup plus importants pour des territoires touristiques (tableau 2). Pour l'année 1999, la richesse foncière uniformisée per capita moyenne variait de $39983 \$$ à $49846 \$$ selon les catégories de municipalités au Québec.

\section{L'ORIENTATION DES CENTRES LOCAUX DE DÉVELOPPEMENT (CLD.) : SOUTIEN À L'ENTREPRENEURIAT VISANT LE DÉVELOPPEMENT LOCAL}

Depuis l'implantation obligatoire des centres locaux de développement (CLD) au printemps 1998, le tourisme peut être

\begin{tabular}{|c|c|}
\hline $\begin{array}{l}\text { TABIEAU } \\
\text { Richesse fonciere } \\
\text { per capia - }\end{array}$ & formisec \\
\hline $\begin{array}{l}\text { Municipalité régionale } \\
\text { de comté }\end{array}$ & R.F.U./capita \\
\hline Les Pays-d'en-Haut & $92151 \$$ \\
\hline Les Laurentides & $81553 \$$ \\
\hline Memphrémagog & $65756 \$$ \\
\hline
\end{tabular}

Source : ministère des Affaires municipales et de la Métropole, 1999 inscrit dans les objets du développement local à l'échelle territoriale de la municipalité régionale de comté (MRC) ou de la communauté urbaine (C.U.) dont le financement est assuré par le gouvernement et les contributions des municipalités locales.

L'offre touristique soutenue par cette filière peut être davantage articulée autour d'un produit, d'une spécialité ou même d'un événement et sous-tendue par la construction d'une identité touristique locale qui dépasse la simple identité administrative. Le projet de développement local fournit l'instrumentation qui permet de construire un projet de territoire touristique tel que Chazaud le propose (tableau 3).

Évidemment, la perspective du développement, appliquée au tourisme, permet de s'adresser simultanément à la population locale et régionale pour la faire participer, sinon à l'offre du produit, du moins à la construction de l'expérience touristique qui peut être proposée et qui, de plus en plus, est gage de réussite dans un environnement marqué par la concurrence.

\section{LA RÉORGANISATION MUNICIPALE : PERSPECTIVE 2000}

\section{LES FONDEMENTS}

Le projet de réorganisation municipale est fondé sur le constat de divergences drastiques des pratiques, surtout des villescentres par rapport à celles de leurs banlieues, même dans les agglomérations éloignées et de petite taille. En 2000, la ministre des Affaires municipales et de la Métropole remet en cause les préceptes de

\begin{tabular}{|c|c|}
\hline \multicolumn{2}{|c|}{ TABLEAU } \\
\hline $\begin{array}{l}\text { Éléments nécessaires à la construction } \\
\text { d'un projet }\end{array}$ & Difficultés et contraintes \\
\hline $\begin{array}{l}\text { Une identité du groupement } \\
\text { de communes }\end{array}$ & $\begin{array}{l}\text { La difficulté est de passer d'une identité } \\
\text { administrative à une identité touristique, } \\
\text { d'une identité communale à une identité } \\
\text { intercommunale }\end{array}$ \\
\hline $\begin{array}{l}\text { Une identité et une notoriété } \\
\text { reconnaissables par le touriste }\end{array}$ & $\begin{array}{l}\text { Le développement touristique doit pren- } \\
\text { dre appui sur une identité territoriale et } \\
\text { transmettre aux touristes une image du } \\
\text { territoire perceptible jusque dans une } \\
\text { offre structurée et ciblée }\end{array}$ \\
\hline Un projet de territoire cohérent & $\begin{array}{l}\text { Le projet de territoire doit conduire à or- } \\
\text { ganiser, à valoriser, à préserver, à label- } \\
\text { liser les espaces, en évitant de les trans- } \\
\text { former en simple parking à touristes }\end{array}$ \\
\hline Un système de valeurs commun & $\begin{array}{l}\text { Les acteurs locaux, pour à la fois préser- } \\
\text { ver l'identité du territoire et le structurer } \\
\text { en fonction d'un positionnement, doivent } \\
\text { adhérer à un système de valeurs commun } \\
\text { (respect du patrimoine, volonté de mo- } \\
\text { derniser l'offre sans dénaturer les sites, } \\
\text { etc.) }\end{array}$ \\
\hline $\begin{array}{l}\text { Une synergie entre } \\
\text { collectivités locales }\end{array}$ & $\begin{array}{l}\text { Des types de partenariats doivent être } \\
\text { mis en place entre les diverses collec- } \\
\text { tivités locales et dépasser la logique de } \\
\text { l'office du tourisme ou du syndicat d'ini- } \\
\text { tiative }\end{array}$ \\
\hline
\end{tabular}


l'autonomie locale dans l'exercice de la mission d'allocation qui consiste à fournir au prix coûtant les services dont les contribuables choisissent de se doter.

Plusieurs arguments viennent appuyer la tendance réformiste. D'abord, l'évolution linguistique amènerait dans vingt ans le français à n'être parlé à la maison que par 49,7\% de la population de l'île de Montréal ; par conséquent, plusieurs municipalités qui la desservent réclameraient progressivement un statut de ville bilingue. Ensuite, la forte coexistence du monde rural et du monde urbain à l'échelle du territoire québécois, y compris même celui de la région métropolitaine de Montréal, exige un partage des fonctions et des charges plus articulé que celui qui a actuellement cours dans la plupart des MRC et des communautés, en particulier en matière d'aménagement du territoire, de transport en commun, de planification de la gestion des matières résiduelles, de logement social et de développement économique.

\section{LES MODES ORGANISATIONNELS}

La réorganisation du secteur municipal, telle que proposée, repose d'abord sur la création d'une nouvelle instance, soit la communauté métropolitaine (C.M.), pour les territoires métropolitains de Montréal, de Québec et de l'Outaouais. Ces territoires métropolitains, plus étendus que les territoires des communautés urbaines actuelles, correspondent approximativement à ceux des régions métropolitaines de recensement concernées. Cette nouvelle instance se voit attribuées des compétences, largement orientées vers les services aux personnes physiques et morales, particulièrement pour le transport en commun, le logement social, le développement économique et les équipements, les infrastructures, les services et les activités à caractère métropolitain. En ce qui concerne la communauté métropolitaine de Montréal, le groupe de travail a recommandé d'abord l'adoption d'une courte liste d'équipements, d'infrastructures, de services et d'activités à caractère métropolitain. Cependant, le Conseil de la communauté, composé de vingt-huit membres, pourra réviser la liste dès 2001.

Les compétences des trois communautés métropolitaines permettront donc, dès 2001, de soutenir le secteur du tourisme,

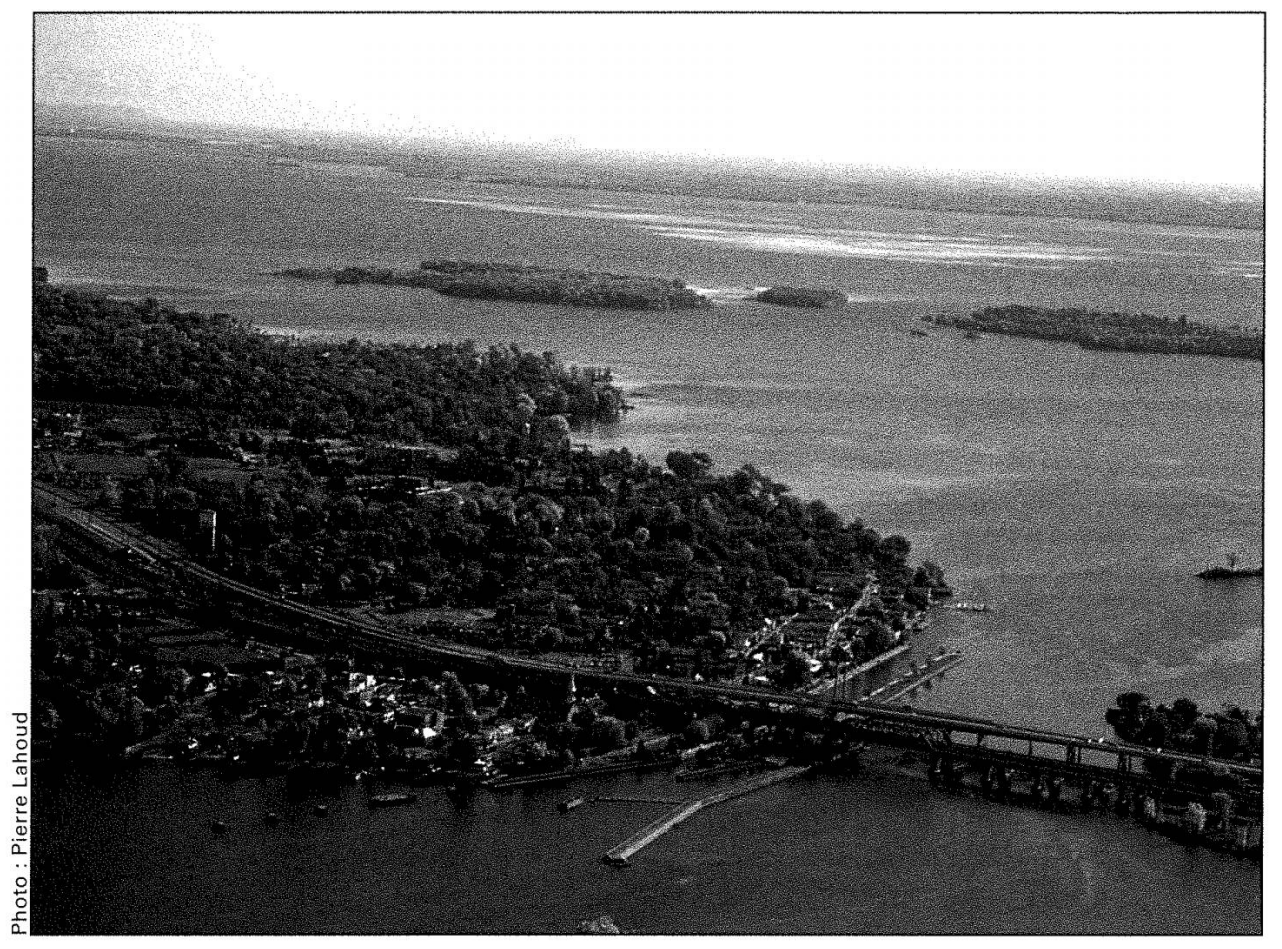

Vue aérienne de Sainte-Anne-de-Bellevue

tant sous l'angle du développement économique général, selon l'importance de la composante touristique que l'on veut y introduire, que sous l'angle des attractions et des activités.

À l'extérieur des communautés métropolitaines, on a confié un mandat de recommandation aux MRC relativement au partage du coût des équipements, des activités et des services à portée supralocale. Même si les travaux ne semblent pas avoir été partout fructueux, il devrait y avoir adoption prochaine et entrée en vigueur d'un décret spécifiant la liste des services et des activités à portée supralocale dans les MRC.

Enfin, la réorganisation du secteur municipal implique des fusions dont on discute actuellement le nombre, l'ampleur et l'échéance. Tous les indices laissent supposer que les mises en commun souhaitables pour les villes-centres qui ne seraient pas couvertes par l'exercice des compétences des communautés métropolitaines ni des MRC quant au corpus supralocal seront réalisées par des fusions des territoires contigus concernés. Une volonté gouvernementale semble s'exprimer pour assurer l'importance et la pérennité de la desserte supralocale autrement que par la conclusion d'ententes intermunicipales volontaires et négociées.
Qu'il s'agisse de municipalités locales redéfinies, de MRC renforcées ou de communautés métropolitaines, les nouvelles structures permettront de relier les dessertes; par exemple, de nouveaux aménagements ne pourront être inscrits au schéma que dans la mesure où on y planifiera le transport en commun.

Les projets touristiques représentent d'opportuns vecteurs de développement des interrelations dans les dessertes et la solidarité régionale.

Les grands projets régionaux, malgré les risques de dérapage qu'ils comportent, restent des occasions privilégiées de développement de la solidarité. Il s'agit là d'une des principales raisons qui militaient en faveur du projet des jeux olympiques d'hiver. Ce projet pouvait mobiliser toute la communauté régionale de Québec telle que décrite plus haut puisque les sites olympiques prévus s'étendaient à l'ensemble des territoires et même au-delà. Si les leaders régionaux et l'ensemble de la population avaient profité de l'occasion pour développer un sentiment d'appartenance régional, ce projet aurait pu être un succès même pour les couches sociales les moins favorisées car il sous-tendait le dévelop- 
pement de l'industrie touristique, secteur économique où nombre d'emplois sont accessibles à tous (Villeneuve et Vachon, 1999 : 58).

\section{LES EFFETS PRÉVISIBLES SUR L'OFFRE TOURISTIQUE}

Quels que soient leurs découpages territoriaux, leurs modalités de fonctionnement, surtout politiques, et l'échéance de leur entrée en vigueur, les changements qui découlent de la réorganisation du secteur municipal seront majeurs et ils affecteront de façon importante la structuration de l'offre touristique locale et régionale.

\section{LA FIN DE L'EXTRÊME FRAGMEN- TATION ET DE LA CONCURRENCE INTERMUNICIPALE EN MATIÈRE TOURISTIQUE}

Les regroupements municipaux dont l'échéance pourrait être aussi rapprochée que le $1^{\text {er }}$ janvier 2002 permettront de définir, au sens touristique, des « pays», des terroirs locaux dont les frontières ne seront plus handicapées par de multiples découpages administratifs. Ils favoriseront l'émergence ou le renforcement de produits touristiques correspondant à la segmentation croissante de la demande par des touristes de plus en plus expérimentés et exigeants et aussi de plus en plus sollicités par des territoires et des produits concurrentiels à l'échelle internationale.

\section{L'ÉMERGENCE ET LE DÉVELOPPE- MENT D'UN TOURISME VÉRITA- BLEMENT MÉTROPOLITAIN}

À Montréal, le mandataire du gouvernement, M. Louis Bernard, propose dans son rapport d'octobre 2000 de remplacer 1'actuelle Communauté urbaine de Montréal par une nouvelle instance appelée Ville de Montréal, dont le territoire couvrirait l'île de Montréal en entier. Il s'agit là d'une proposition qui va au-delà d'une dénomination institutionnelle. En effet, l'actuelle Communauté urbaine de Montréal est une instance dépourvue tant de représentation directe des citoyens (sans élection au suffrage universel) que de pouvoir de taxation directe. La future Ville de Montréal, qui lui succédera pour le même territoire de l'île de Montréal, sera pourvue de la représentation et de la taxation, et elle

bénéficiera aussi de compétences élargies par rapport à celles de l'actuelle Communauté urbaine.

On peut donc s'attendre à l'émergence de partis politiques pour cette nouvelle instance et à la reconnaissance rapide d'enjeux centraux qui seront représentés par la nouvelle ville-centre à la Communauté métropolitaine, laquelle comprend la RiveSud et la Rive-Nord. À cause de sa place dominante, la nouvelle ville-centre aura tout intérêt à définir des enjeux métropolitains dont on prévoit déjà que le développement économique en général et l'activité touristique en particulier constitueront des assises.

\begin{abstract}
Le développement économique comprend plusieurs types de développement, notamment le développement industriel, le développement touristique et le développement commercial. La nouvelle ville aurait une compétence générale en la matière.
\end{abstract}

Chaque type de développement, pour avoir le maximum d'impacts, devrait faire appel à des partenariats différents entre la nouvelle ville, les arrondissements et les milieux d'affaires concernés » (Regroupements municipaux dans la R.M.M, 2000 : 11).

Dans cette perspective, Montréal pourrait évoluer de pratiques de tourisme urbain vers la définition d'une offre touristique véritablement métropolitaine, d'autant que les grands équipements et les attractions seront placés sous la gestion de la nouvelle Ville de Montréal et financés en partie par la Communauté métropolitaine de Montréal, comme le prévoit la loi instituant cette instance.

Ce repositionnement de Montréal parmi les grandes métropoles touristiques internationales d'une taille de population de 1,8 million à 3,5 millions, selon que l'on considère la nouvelle Ville ou la Communauté métropolitaine, ne se fera pas au détriment du tourisme de quartier. Au contraire, les futurs vingt-sept arrondissements prévus au rapport Bernard pour l'île de Montréal s'articuleront autour de l'ensemble des services de proximité et constitueront le lieu idéal d'épanouissement du tourisme de quartier.
Si plusieurs experts et acteurs considèrent que le projet de réorganisation municipale aurait pu revêtir des formes institutionnelles plus simples et plus performantes et qu'il aurait dû viser des économies tangibles au plan fiscal, il n'en demeure pas moins qu'il présente une pertinence incontestable quant au développement du tourisme au Québec. Le tourisme de quartier à Montréal, le tourisme régional et le tourisme métropolitain en constituent les principaux bénéficiaires.

Danielle Pilette, Ph.D., professeure au Département d'études urbaines et touristiques de l'UQAM, est directrice du baccalauréat en gestion du tourisme et de l'hôtellerie et de la maîtrise en gestion et planification du tourisme.

\section{BIBLIOGRAPHIE}

Chazaud, P. (1998), « Développement touristique et intercommunalité de projet », Les Cahiers Espaces, $\mathrm{n}^{\circ} 55$, mars, p. 84.

Conférence Québec-municipalités (1978), La revalorisation du pouvoir municipal. Réforme fiscale ; 1. Fondements et principes, Québec, p. 26.

Ministère des Affaires municipales (1996), Prévisions budgétaires des municipalités locales.

Ministère des Affaires municipales et de la Métropole (1999), Prévisions budgétaires des organismes municipaux.

Ministère des Finances, (1996), Fiscalité et financement des services publics, Oser choisir ensemble. Le secteur local au Québec : organisation et fiscalité, Québec, Les Publications du Québec, p. I.

Regroupements municipaux dans la R.M.M. (2000), Recommandations du mandataire, octobre, p. 11.

Villeneuve, Paul, et Frédéric Vachon (1999), «Intégration territoriale dans la région de Québec », dans G. Mercier, C. Bélanger et M. Bédard, L'avenir municipal, Québec, Éditions Sylvain Harvey, p. 58. 